\title{
The Africa Regional SGBV Network Learning Brief Series: Learning updates from Uganda (Brief \#6)
}

Population Council

Follow this and additional works at: https://knowledgecommons.popcouncil.org/departments_sbsr-rh

Part of the Demography, Population, and Ecology Commons, Domestic and Intimate Partner Violence Commons, Family, Life Course, and Society Commons, and the International Public Health Commons How does access to this work benefit you? Let us know!

\section{Recommended Citation}

"The Africa Regional SGBV Network Learning Brief Series: Learning updates from Uganda (Brief \#6)." Nairobi: Population Council, 2016. 
Population Council

The Africa Regional SGBV Network Learning Brief Series

Fostering a Multisectoral Response to SGBV in Humanitarian Settings

\section{Learning Updates from Uganda (Brief \#6)}

Sexual and gender-based violence (SGBV) in humanitarian contexts is recognized by the UN Refugee Agency (UNHCR) as a global concern and as a priority in its programming. Crisis-affected populations often face multiple forms of SGBV at multiple time periods (during conflict, during flight from conflict, and within post-conflict settings). The Africa Regional SGBV Network, set up in 2006, is addressing SGBV in humanitarian contexts in East and Southern Africa. This Learning Brief is one in a series of two that share critical findings on effective responses to the issue in Uganda.

\section{Screening for SGBV in Humanitarian Settings in Uganda}

Evidence is growing in sub-Saharan Africa that routine screening for SGBV can help detect survivors of SGBV and help them to access comprehensive services. However, these studies have mostly been conducted among general populations and there is sparse evidence for the feasibility of SGBV screening interventions in humanitarian contexts.

Under the Africa Regional SGBV Network, the Population Council and Africa Humanitarian Action (AHA) collaborated with a range of partners (including the Uganda Refugees Department (Office of the Prime Minister), the Child Health and Development Centre, Kenyatta National Hospital, and UNHCR) to assess the feasibility of implementing SGBV screening and referral protocols in a humanitarian context. The research took place in health facility settings in Rwamwanja Refugee Settlement in Kamwenge District, Western Uganda. The settlement is home to around 60,000 refugees, most of whom are from the Democratic Republic of Congo.

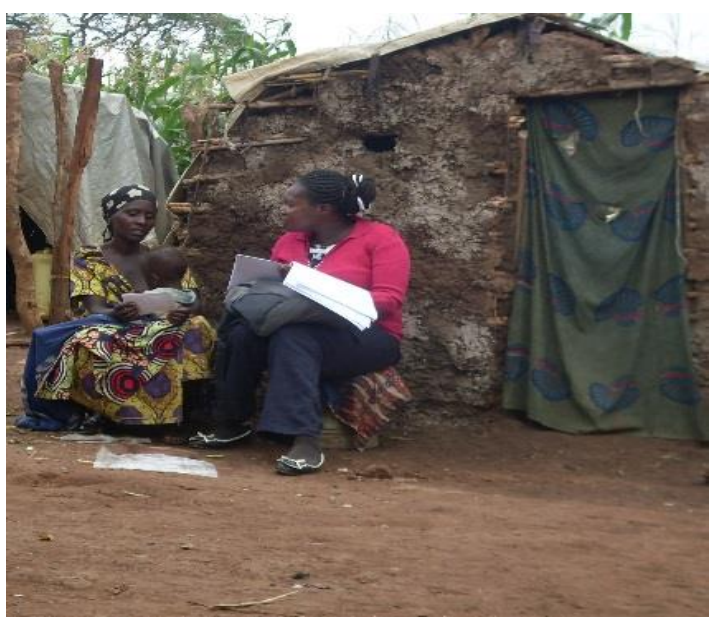




\section{What we're learning}

In the study, trained providers in two health facilities screened female clients routinely for current exposure to intimate partner violence and lifetime exposure to non-partner sexual violence. Those identified were referred for further care. Key findings included the following:

Survivors were willing to disclose SGBV

- $10 \%$ of those screened disclosed that they had experienced some form of SGBV

- Of the $10 \%$, a third disclosed lifetime exposure to non-partner sexual violence, and $84 \%$ disclosed current exposure to intimate partner violence

Providers achieved high rates of SGBV screening and survivor referral

- 8,462 clients were screened in the two health facilities in five months

- $96 \%$ of survivors were referred for comprehensive SGBV care

There were good rates of referral adherence

- $63 \%$ of referrals went for further SGBV care: this is an adherence rate $23 \%$ higher than in a similar study amongst the general population

- More than half of the referred survivors who did not come for SGBV care were screened at a health facility which did not have SGBV services available on site. This systemic barrier to referral adherence is resolvable.

\section{Survivor satisfaction with SGBV services was high}

- [Y]ou can't go telling people you have a problem unless they ask you. ... I have never told anybody about my rape since no one has ever asked me about it. Today, I had a chance for the first time to pour it out and cry about this terrible incident. I am fine now... I was very happy with the services and care that I got from the counselor...This was my first time of pouring out what has been in my heart for a very long time-something I have never talked to anybody about. It was such a big relief. SGBV Survivor, Maternity Department 
Provider satisfaction with implementing screening protocols was high

- [Screening] takes some time...but the truth is, time taken [today] while you are assisting someone will save your time tomorrow, because you will get the correct diagnosis. If you don't take time, you will [prescribe] Panadol, and tomorrow, the client will come back because the problem was not what you treated-it was psychological. So, it is better to take those two minutes [to] address...the real problem. Then, you will save more time. Provider, ART Department

SGBV screening and referral were highly acceptable to those involved

- The screening intervention led survivors and providers to share ideas about related initiatives that could be implemented alongside screening to comprehensively address SGBV. Their ideas are considered in our recommendations.

\section{What we recommend}

The relatively higher screening, disclosure, referral, and referral adherence rates under the study reveal a real need and demand for SGBV screening in humanitarian settings.

The study led to four key recommendations for strengthening SGBV screening protocols, and for addressing gaps in SGBV programming more broadly in humanitarian contexts in the region:

1. Screening must be accompanied by referrals to services available at the same site - this will ensure better referral adherence and greater access to comprehensive SGBV care.

2. SGBV screening and services could be integrated into reproductive health care and general health service delivery, where possible, so that SGBV screening becomes a standard procedure. This would be particularly useful in humanitarian settings were the occurrence or experience of SGBV is likely to be high.

3. Increase the number of trauma counsellors and improve the training curricula for counsellors. Training curricula need to address the specific, complex SGBV realities found in humanitarian contexts (e.g., rape-related pregnancy, and trauma resulting from the experience of multiple violence forms simultaneously).

4. Involve men in the response to SGBV. Many female survivors and health providers highlighted the importance of addressing SGBV in the lives of men, and of involving men in SGBV interventions for women. Suggestions included couple counselling, screening and counselling for men and interventions at the community level to reach both men and women. 
For a more detailed report:

http://www.popcouncil.org/uploads/pdfs/2016RH_ScreeningSGBV-Uganda.pdf

Since 2006, the Africa Regional SGBV Network has worked to build effective responses to SGBV in low-resource settings, focusing on those who have experienced violence, as well as on violence prevention. From 2014-2017, the network is addressing the needs of two vulnerable populations - children and refugees.

(c) 2016 The Population Council, Inc.

Network Contact:

Population Council

General Accident Insurance House

Ralph Bunche Road

Nairobi, Kenya

$\mathrm{T}:+254202713480$

E: info.nairobi@popcouncil.org
AHA Contact:

Muyenga Ggaba Road Plot 4285

Block 224

P.O. Box 7730

Kampala, Uganda

$\mathrm{T}:+256414510452$

E: uganda@africahumanitarian.org

We gratefully acknowledge the support of the Regional Team for Sexual and Reproductive Health and Rights, Embassy of Sweden, Lusaka, Zambia. 